\title{
MARS Glass: A Smart Personal Protective Wearable
}

\author{
Muthucumaru Maheswaran'1 and Maiuri Maheswaran ${ }^{2}$ \\ McGill University \\ Montreal, Canada
}

$\mathrm{W}$

earable computing is a fast evolving segment of computing that includes smart watches, head mounted wearables such as Magic Leap headsets, Microsoft Hololens, and VR goggles from various vendors. In this report, we present ideas for a smart wearable device that also doubles as a virus protection device. Instead of using the filtering approach that is predominantly used by virus protection equipments such as face masks, we propose to use a computational approach where the device maintains an awareness of the real-time virus spread and use that information to steer the wearer away from the virus. As the wearable has a head enclosing design, viral infection can only happen through the air that is inhaled by the wearer. The objective of the smart wearable is to maintain a repository for clean air and switch the operating modes between stored and fresh air modes depending on the environmental conditions. It can augment this basic operating procedure by recycling the exhaled air to maximize it operating capacity (i.e., time duration for which it could supply the wearer with safe air) and by cleaning the stored air using UVC to further reduces the chance of infection. To maintain an awareness of the virus spread in the environment, the smart wearable will rely on an edge computing framework that will be distributed to cover areas frequented by people. The smart wearable will have modular design so that it can be reconfigured to add or subtract functionality that the wearer wants for a particular situation so that the design remains relevant even after the virus threat recedes.

\section{Smart Personal Protective Wearable}

We are engaging with many smart gadgets in our day-to-day lives to the extent that they are becoming almost an indispensable part of many of our activities. It is widely believed (and marketed) that the smart versions of the gadgets are improving the quality of our lives while making the whole ecosystem more efficient. For example, smart phones are considered to be very productive by many people because it supports many different forms of communication and communication related applications (e.g., calendaring) in a single device. Because smart

\footnotetext{
1 muthucumaru.maheswaran@mcgill.ca

2 maiuri.maheswaran@mail.mcgill.ca
} 
versions of the gadgets are expensive, they cannot be as uni-functional as their lower quality counterparts. Thus, the smart gadget needs to provide larger number of services in addition to a higher quality of experience. For instance, smart watches tend to encroach into the territory of smart phones, fitness trackers, and even medical devices in their quest to expand their appeal to the customers. With a larger portfolio of services, smart gadgets are also supported by multiple and diverse stakeholders ranging from social networking services to healthcare providers.

In this report, we propose a design for a smart personal protective wearable called MARS (Mobile Air Repository System) Glass. The objective is to create a design at the crossroads of many industries so that it would be propelled by many players. For instance, traditional watch manufacturers are dwarfed by others in the smart watch industry. A wide ranging array of players from computer manufacturers to fashion retailers are involved in creating smart watches. Similarly, by creating a design for a smart personal protective wearable that attracts many players from variety of industries, we can accelerate its development and deployment. Despite a broad-based design, the smart personal protective wearable will primarily function as a protective device that will also support other services.

The smart personal protective wearable is a device that allows the wearer to avoid getting infected by the virus, just like the personal protective equipment such as medical masks or respirators. The masks and respirators work by filtering the viruses and reducing the probability of humans getting infected by the virus. Because the masks and respirators are exposed to the virus, there is a problem in reusing them or even taking them off. The smart personal protective wearable, on the other hand, is designed to avoid the virus itself. This way the wearable does not harbour the pathogens and minimal hazard is present in taking it off or putting it on. As the wearable is avoiding the virus, it remains reusable as well.

The basic idea behind MARS is to treat air as a reusable commodity and avoid immediate reuse of it. That is, a MARS Glass should not draw in the air that has been exhaled by another person wearing a MARS Glass or not. The MARS Glass wearer breathes the air that has been drawn in by the device. By deciding whether it is safe to draw in a particular volume of air, the MARS Glass controls the air that is inhaled by the wearer. To provide an uninterrupted supply of air to the wearer, the MARS Glass needs to store a sufficient supply of safe (free of pathogens) air that can be inhaled during the time intervals where it cannot draw in air from the surroundings. It is the responsibility of the MARS Glass to map the surroundings and intelligently switch between the fresh air (air that is drawn from the surroundings) and stored air (air that is in the MARS Glass storage that was previously drawn in) modes. To switch between the fresh air and stored air modes, the MARS Glass needs to model the virus dispersal in the surroundings and determine whether it is safe to draw in air in a given location at that instant.

The MARS Glass idea shares a common underlying principle with social distancing plus face masks. By increasing the distance between people, social distancing reduces the infection 
rates (this is validated by the effectiveness of social distancing [1]) because increased distance makes direct virus transmission less likely. This principle also applies to face masks. Taken together, social distancing and face masks limit the chance of one person inhaling the pathogens that are exhaled by another person. With MARS Glass we are asking the question whether this objective of avoiding immediate air reuse can be achieved computationally.

\section{Key Requirements for Smart Protective Wearables}

MARS Glass has two categories of requirements. The first set of requirements comes from it being a smart wearable. The second set of requirements come from it being a smart wearable.

To protect the wearer, the MARS Glass must be effective in significantly reducing the risk of the wearer getting infected with COVID-19. They should be able to carry on with their daily routines without worrying about the risk of infection. Another requirement that differentiates MARS Glass from other PPE, such as surgical masks and N95 respirator masks, is that the effectiveness of the MARS Glass should not depend on communal use. The MARS Glass should be effective individually without relying on mass acceptance. MARS Glass should be reusable and should be cost-effective over a period of usage.

As the MARS Glass is a smart device, it falls in a different cost bracket than other non-smart wearables. The smart features bring the next set of requirements. As a device that covers the head, MARS Glass is a prime candidate for supporting emerging technologies such as augmented reality or mixed reality. Many headsets proposed and manufactured (e.g., Magic Leap and Microsoft Hololens) for these technologies have been expensive and bulky. By supporting augmented or mixed reality, MARS Glass gives itself the room to have more computing power; some of which could be used for virus protection services. In addition to the augmented or mixed reality functions, MARS Glass needs to provide high fidelity personal speakers (i.e., speakers inside the headwear that would not disturb others). Using these features many smart phone based applications can use MARS Glass as a display device. For example, media streaming, games, news, and messaging services can be hosted in the MARS Glass.

Another set of the requirements is to make the gadget truly reconfigurable much like the modular smartphone (Project Ara). With a headwear that can be reconfigured in different ways, the gadget can remain relevant even after the virus threat has receded.

\section{Personal Protective Equipments: A Quick Survey}

The face masks is the main type of PPE that is playing a critical role in fighting the COVID-19 virus. These masks have been a staple in the war against airborne flu pandemics; their usage date back to the early 20th century (e.g., Spanish Flu). 
There are three types of face masks in widespread use: surgical masks, cloth face coverings and respirators. Surgical masks and cloth face coverings are similar in principle. The surgical mask is a loose fitting mask that captures large respiratory droplets (droplets that are greater than 60 microns in diameter) that are emitted by the wearer [2]. By capturing these droplets, it prevents them from seeping into the environment and infecting others. To a certain degree, it also protects the wearer from droplets emitted by others.

It is important to note that surgical masks only protect against large respiratory droplets and not small respiratory droplets. Both size droplets can contain SARS-CoV-2 and infect individuals but the small droplets can sediment in the alveoli sacs of the lungs and can cause lethal symptoms associated with the disease. Surgical masks have been shown to capture $75 \%$ of emitted respiratory droplets [3]. Face coverings created using cloth also offer similar protection as the surgical; although they are considered by many to be less effective based on their construction.

The last category is the respirator which offers maximum protection against both small and large respiratory droplets. Respirators can be classified according to the method of moving air: active or passive. Passive respirators, such as the N95 respirator, require lung power to move air through the filter. The N95 can filter $95 \%$ of the airborne particles; including particles that are as small as 5 microns in diameter. Unlike the surgical and cloth masks, the N95 mask perfectly covers the air pathways and maximize protection but this, in itself, has limitations as it causes discomfort to young children and those with facial hair. Moisture and heat buildup is a possibility with face masks. The other type of respirator is the active respirator which is battery powered. One such example is PAPR (powered air-purifying respirator) that has a battery-powered air filtering system alongside a face hood. The PARP has a higher filtering capacity than the N95 respirator; with a 99.7\% efficiency. A major disadvantage of the PARP is its complexity which mandates formal training obtained only by healthcare professionals [4].

To be effective, surgical masks and cloth coverings need mass compliance. With anger erupting over mandatory mask-wearing policies, it is becoming painstakingly obvious that this adherence may not be feasible in the long-term. Another issue that is commonly overlooked is the barriers the mask imposes on the deaf and hard-of-hearing who depend on reading lips to interpret others [5]. The masks obstruct the lips so this ability is hindered.

It is estimated that every month, the world needs 89 million medical masks. Many of the PPEs, such as the N95 mask and the surgical mask, are made of plastic and are single-use. They are also made of polyethylene which is a very dense thermoplastic that is non-biodegradable and non-recyclable. Their constant usage has led to an increase in ocean pollutions as reported by conservation groups. The mask that contributes less to pollution is the home-made mask. However, those are the masks that offer the user the least amount of protection as they use the least amount of layers [6]. 


\section{The Virus Dispersal Model}

The MARS Glass relies heavily on a computing model that is able to map the movements of respiratory particles emitted by infected individuals. The computing model takes into consideration the direction of air currents, type of space, location of people the people, their movements, etc. The objective of the computing model is to track the chance of finding virus in the air. The MARS Glass would use this chance to decide whether it should use stored air or fresh air. In addition to the air quality, MARS Glass would also track the movements of the wearer to compute a plan for air supplies. Highly congested places without safe air pickup locations will be marked as localities that the wearer should avoid.

We want a virus dispersal model that would allow us to estimate virus residency in the air using parameters we can measure without intrusive examinations. For instance, COVID-19 models from GaTech show that the likelihood of an infected person infecting others in a given area increases in proportion to the size of the event as well as the number of active cases within the area. For example, if a country has 1000 cases of COVID-19, there is a $3 \%$ chance that someone who is infected will show up to a 10,000 person event. However, if there are 10,000 cases within a country, there is a $26 \%$ chance that an infected individual will attend the same event [7]. The infected individual can be symptomatic or asymptomatic.

We need to at least solve the following problems to get a virus dispersal model to use with the MARS Glass. We need to detect people and their activities in the area being modelled. That is, we want to detect the number of people, their residency times, the activities they engaged in while in the area. For instance, some people would have just passed by the area which would pose a different virus emission chance than those people staying and talking in that area. We want to capture the activities using means that would identify the individuals without facial recognition or video feeds. The techniques that use WiFi perturbations as means to track for human activity tracking is an idea that can be used mainly because they infringe the least on privacy.

Various studies have been done to quantify the amount of viral particles that are in a cough or sneeze or the viral particles that are emitted every time one breathes or talks. A sick person can emit a greater volume of aerosols and thus a greater number of droplets. These droplets from both sneezes and coughs can contain up to 2 hundred million viral particles [8]. The particles emitted by sneezing travel at a speed of about $20 \mathrm{~m} / \mathrm{s}$ while particles emitted by coughing travel at about $10 \mathrm{~m} / \mathrm{s}$. Breathing and talking, on the other hand, can generate around 50 particles/ sec. The particles emitted by all forms of methods are variable in size and range between 0.001 and 1000 microns in diameter [9]. The large particles, which are typically greater than 1000 microns, are subjected to the force of gravity and fall to the ground after 30 seconds [10].

However, the smaller particles which are refer to as micro droplets and have a size of 10 microns, can drift in a cloud of gas and remain for up to 20 mins [10]. This drifting, in a 
stagnant room, is random. In the case of an open room, it is subjected to the movement of the wind which is $\mathrm{m} / \mathrm{s}$ and $3 \mathrm{~m} / \mathrm{s}$, outdoors $1 \mathrm{~m} / \mathrm{s}$ horizontal and $0.1 \mathrm{~m} / \mathrm{s}$ vertically in stable air [11]. If there is an air source and the emitter of the viral particles is in the path of this source in an enclosed space, the viral particles will be highly concentrated along the path of the air source. This was observed in a Guangzhou restaurant where clients sitting in the path of an air conditioner were all infected with COVID-19 [12]. However, in an open space, the wind helps to flush the room from viral particles

The virus dispersal model we want to create is one that is specific to a given location. Since the viral particles cannot be measured directly, we need a way of indirectly modelling its residency in the air and track its variation with time.

\section{MARS Glass Design}

As shown in the figure below, the wearable interacts with stationary computers that are distributed throughout the space. The distributed computing system is responsible for tracking the activity occurring within the space. To preserve the privacy of the individuals visiting the area, the distributed system can use WiFi based human activity tracking. The WiFi based human activity tracking can be passive yet it needs to detect the activity of the people; whether they have electronic devices or not. The nodes in the distributed system are stationary and each node is responsible for a specific area. The mobiles nodes are the wearables. The mobile nodes connect with the closest stationary nodes as the individuals roam the areas. By connecting to the closest node, the wearable will be able to contact the node that is responsible for the area and get the real-time updates. The MARS Glass and the distributed computing infrastructure will use edge computing based programming framework (e.g., JAMScript) to create a system that can automatically and rapidly connect to the appropriate edge node.

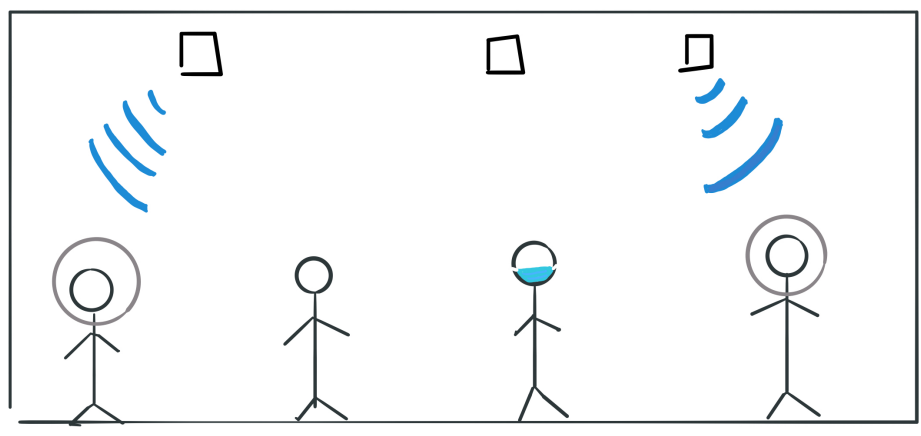

The MARS Glass wearable is a head enclosing bubble helmet [13]. It is fed air from an air storage tank that the wearer would carry as a backpack. The storage tank would hold air in compressed form. The tank is filled with air by a compressor that is run by battery power when the MARS Glass controller decides it is safe to fill up the repository with fresh air. The 
air is released into the bubble helmet so that the wearer does not breath air from his/her surroundings, but the air that is provided by the storage tank of the MARS Glass. The Mars Glass thus determines the air the wearer is breathing. By picking up the air that is least likely to have viral particles, it can minimize the risk to a level below the acceptable threshold. The energy released from the expanding the air that is released from the storage into the helmet can be recouped if the compressor can be run in reverse and the available energy is significant enough to capture.

One of the problems with the simple compress-store-release scheme is the amount of air that should be stored in the tank. To support 10 mins of breathing, we need to carry about 80 litres of air. With a 20x compression, we need a storage tank with 4 litres of capacity. To reduce the bulk of the air storage, we can recycle some of the exhaled air. The exhaled air will have higher levels of $\mathrm{CO} 2$ and moisture. We can remove these elements and mix it with the fresh air and feed it to the helmet. With recycling, we can support a 10 mins breathing time for the wearer with a much smaller tank.

We can use a filter for removing the moisture and $\mathrm{CO}$. While there is no commercial available filter for $\mathrm{CO}$, a group in MIT developed a carbon capture machine that resembles a battery [14]. There must also be a mechanism that extracts the nitrogen so that the oxygen concentration in the container can resemble the concentration seen in normal situations.

Another problem is virus intake into the storage tank. Although the MARS Glass is using a computational approach to minimize the risk of intaking air with viral particles, there is a small chance viral particles can find their way into the storage. We can use UVC radiation to deactivate the viral particles inside the air storage. UVC is one of the three types of UV radiation and has the highest energy. A paper published by Columbia University found that far UVC light, which has a wavelength of $222 \mathrm{~nm}$, can kill coronavirus while not causing damage to human cells. It can kill $99.9 \%$ of airborne viral particles and does not discriminate between different viruses [15].

\section{Example Use Cases}

In this section, we explain several example use cases of MARS Glass. We also describe deployment issues that are related to the use cases.

We expect MARS Glass to be highly helpful for warehouse workers. Currently, companies such as Amazon have invested money in creating bracelets that ensure that employees are standing 6 feet apart from one another. For the bracelet based approach to work, each worker needs to wear the bracelet and when two bracelets come within 6 feet, both bracelets will start beeping [16]. The workers are expected to take the warning cue to distance themselves. The MARS Glass would allow the workers to collaborate in close spaces without worrying about distancing. Because the workers are likely to have assigned stations in warehouses or 
factories, air supply lines can be constructed so that the warehouse will have many air supply stations. The air supply draws fresh safe air from outside. The workers can connect their MARS Glass wearable to the air supply stations and avoid frequent trips for air pickup. In addition to providing a supply of safe air, MARS Glass can be used by the workers to get information on packages (through augmented reality) and other work related actions, which improves their productivity.

Another scenario in which the MARS Glass would prove to be useful is for customer service workers, in particular restaurant workers and customer service employees. It is estimated that more than 5 million restaurant workers have lost their jobs [17]. By wearing MARS Glass, workers can ensure that they don't catch the virus while remaining in contact with the customers. Also, they could use the augmented virtual reality technology to their advantage as a means of tracking which table has been served and the orders and requests of each customer. It is important to note that the MARS Glass can only be used for short intervals of time. Because the restaurant workers are highly mobile, they don't need air supply stations. They can move to locations that will allow safe air pickup.

One of the design goals of MARS Glass is to accommodate necessities beyond the pandemic. One interesting usage is during allergy season. Seasonal allergies, such as pollen allergies, debilitate people. With a modular design, MARS Glass can be augmented with filters that target pollens. Another population that would benefit from the MARS Glass are the farmers. Farmers are routinely exposed to pesticides [18]. MARS Glass can deploy its computational capabilities to determine the pesticide spraying direction, durations, etc based on the wind direction, time of day, etc. The augmented reality feature is quite handy as it allows farmers to keep track of their crops, what are crops are growing in what portion of the land, the status of growth of each crop, etc.

\section{A Path To Realizing MARS Glass}

This document lays down the initial idea for MARS Glass. As the proposed design implies, creating MARS Glass is an ambitious research and development venture. While the design section of this report focused on the technical side of MARS Glass, there are business, social or cultural, ergonomic or fashion sides as well.

Smart wearables is already a significant market. Technologies such as augmented reality, mixed reality are still mainstream, but are expected to be technologies with major disruptive potential. There is a need to study the business side of problem to determine how mixing virus protection changes the interest in smart wearables. In particular, the application of health care regulations to the MARS Glass can be a concern from the business side. Stringent health care regulations can add many more years to a product before it can reach the market, which can create major concerns in the business side. 
There are cultural implications that arise with the MARS Glass. People who practice certain cultures often wear head coverings to signal their devoutness to God. The MARS Glass thus needs to be built to accommodate these needs and give enough space for bulky dressings such as turbans. Other implications include the maintenance of beards; which is a religious observance prevalent for Sikh men. Two Sikh physicians in Canada shaved their breads so that they could wear the N95 mask [19]. This a difficult decision to make and one's religious observance should not be compromised. Thus, the MARS Glass would need to be built to accommodate such observances.

Finally, the last part worth consideration is the fashion aspect. Many consumers judge wearables based on the design and the visual appeal of the device. It has to be modern and sleek. The MARS Glass should not be bulky as this would dissuade many from wearing the device. It should be customizable so that people can have it in custom colours or patterns; which may be appealing to many buyers. Also, the MARS Glass's features, such as augmented reality and messaging, should be designed in such a fashion that complements the viewer and that adds to the experience.

\section{Conclusion}

In this report, we proposed a design for a new type of smart wearable called MARS Glass. The primary use case of the wearable is to be a head enclosing wearable that would protect the wearer from airborne virus particles. The basic concept underlying the MARS Glass is quite simple: carry your own air. This concept is inspired by what is already being practiced in many parts of the world for water - carry your own water - where there are deadly waterborne diseases in those parts of the world.

The challenge with carrying your own air is the difficulty in carrying a lot of it. We can only carry air that is good for 10 to 15 mins of breathing time. This means the gadget needs draw air from the environment sooner or later. We propose to use a distributed computing scheme to create real-time awareness of the virus spread. The MARS Glass uses this awareness to determine whether it should use stored or fresh air at a given location at a given time. We want to make use of the emerging edge computing technologies to create this awareness framework so the data remains local and the services offered by the system remains available at real time.

In addition to its primary role as a virus protective equipment, we want the MARS Glass to be a host to a variety of modern applications including the ones in augmented and mixed reality. This is possible because MARS Glass is a head enclosing wearable. By adding other features besides virus protection, MARS Glass has many factors driving its adoption from both the consumer and producer sides. 
In summary, we present ideas for a new wearable design that would achieve social distancing and masking without putting a physical distance between people. The idea is to enable people to move and engage with their day-to-day actions and by gently nudged by the computer to alter their behaviour to deal with the virus. For instance, take a small break from a crowd to get an air refill.

\section{References}

(1) Chu, Derek, Derek K et al. Physical distancing, face masks, and eye protection to prevent person-to-person transmission of SARS-CoV-2 and COVID-19: a systematic review and meta-analysis. The Lancet, Volume 395, Issue 10242, 1973 - 1987

(2) Cobb, Cynthia. May 26 2020. Healthline. "What is the Best Type of Face Mask for You?", accessed June 2 2020. https://www.healthline.com/health/best-face-mask-2

(3) FDA. "N95 Respirators, Surgical Masks, and Face Masks". https://www.fda.gov/medicaldevices/personal-protective-equipment-infection-control/n95-respirators-surgical-masksand-face-masks

(4) Roberts V. To PAPR or not to PAPR? Canadian Journal of Respiratory Therapy: CJRT = Revue canadienne de la therapy respiratoire: RCTR, 50(3) 87-90

(5) The Canadian Press. CTV Montreal. June 20 2020. "Masks are the worst nightmare of the deaf and hard of hearing who read lips”, accessed July 12020. https://montreal.ctvnews.ca/ masks-are-the-worst-nightmare-of-the-deaf-and-hard-of-hearing-who-read-lips-1.4992889

(6) Clark, Nick. Al Jazeera. 2 Jul 2020. "Face masks, gloves and a new breed of plastic pollution”, accessed Jul 4 2020. https://www.aljazeera.com/indepth/features/face-masksgloves-breed-plastic-pollution-200701154052002.html

(7) Maggiacomo, Taylor and Michael Greshko. March 19 2020. "See why keeping groups small can save lives in the era of COVID-19”, accessed July 92020.

(8) Bardi, Jason Socrates. LiveScience. June 14 2009. “The Gross Science of a Cough", accessed July 9 2020. https://www.livescience.com/3686-gross-science-cough-sneeze.html

(9) Guzman, M. Bioaerosol Size Effect in COVID-19 Transmission. Preprints 2020, 2020040093.

(10)Ningthoujam R. COVID 19 can spread through breathing, talking, study estimates [published online ahead of print, 2020 May 8]. Curr Med Res Pract. 2020;10(3):132-133.

(11) Identifying airborne transmission as the dominant route for the spread of COVID-19. Renyi Zhang, Yixin Li, Annie L. Zhang, Yuan Wang, Mario J. Molina. Proceedings of the National Academy of Sciences Jun 2020, 117 (26) 14857-14863 
(12) Lu J, Gu J, Li K, et al. COVID-19 Outbreak Associated with Air Conditioning in Restaurant, Guangzhou, China, 2020. Emerging Infectious Diseases. 2020;26(7):1628-1631.

(13)Easton, John and Matt Wood. UChicago Medicine. March 25 2020."Helmet-based ventilation is superior to face mask for patients with respiratory distress”, accessed June 12 2020. https://www.uchicagomedicine.org/forefront/patient-care-articles/helmet-basedventilation-is-superior-to-face-mask-for-patients-with-respiratory-distress

(14)Chandler, David. MIT News Office. October 24 2019. "MIT engineers develop a new way to remove carbon dioxide from air”.http://news.mit.edu/2019/mit-engineers-develop-newway-remove-carbon-dioxide-air-1025

(15) Buonanno, M., Welch, D., Shuryak, I. et al. Far-UVC light (222 nm) efficiently and safely inactivates airborne human coronaviruses. Sci Rep 10, 10285 (2020).

(16)Palmer, Annie. CNBC. Jun 16 2020. "Amazon is testing a wearable device that lights up and beeps when warehouse workers get too close to each other", accessed Jun 202020. https://www.cnbc.com/2020/06/16/amazon-tests-wearable-social-distancing-device-forwarehouse-workers.html

(17) Catrodale, Jelisa. VICE. May 9 2020. "More than 5 Million Restaurant Workers Lost Their Jobs in April”, accessed June 4 2020. https://www.vice.com/en ca/article/qj4d7m/morethan-5-million-restaurant-workers-lost-their-jobs-in-april

(18)Damalas CA, Koutroubas SD. Farmers' Exposure to Pesticides: Toxicity Types and Ways of Prevention. Toxics. 2016;4(1):1. Published 2016 Jan 8.

(19)Elassar, Alaa. CNN, May 16 2020. “Two Sikh doctors shaved their beards, a pillar of their religion, to treat Covid-19 patients, But comment groups say there are alternatives”, accessed June 10 2020. https://www.cnn.com/2020/05/16/health/sikh-doctors-beardscoronavirus-trnd/index.html 